Kragujevac Journal of Mathematics

Volume 42(4) (2018), Pages 619-630.

\title{
PSEUDO CONHARMONICALLY SYMMETRIC SPACETIMES
}

\author{
F. ÖZEN ZENGIN ${ }^{1}$ AND A. YAVUZ TAŞCI ${ }^{2}$
}

\begin{abstract}
In the present paper, firstly, the definition of pseudo conharmonically symmetric Riemannian manifold is given. In the second section, some theorems about these manifolds are proved. In the third section, pseudo conharmonically symmetric spacetime is investigated. Under some special conditions, we examine the properties of this spacetime.
\end{abstract}

\section{INTRODUCTION}

Because of the important role in differential geometry of symmetric spaces, in the beginning of the last century, Cartan [4] initiated Riemannian symmetric spaces and obtained a classification of these spaces. Let $(M, g)$ be an $n$-dimensional Riemannian manifold with the Riemannian metric $g$ and the Levi-Civita connection $\nabla$. If the condition $\nabla R=0$, where $R$ is the Riemannian curvature of a Riemannian manifold, then this manifold is called locally symmetric, [4]. In every point $P$ of this manifold, this symmetry condition is equivalent to the fact that the local geodesic symmetry $F(P)$ is an isometry [12]. The class of Riemannian symmetric manifolds is very natural generalization of the class of manifolds of constant curvature. The notion of locally symmetric manifolds have been studied by many authors by extending several manifolds such as conformally symmetric manifolds [6], recurrent manifolds [26], conformally recurrent manifolds [2], conformally symmetric Ricci-recurrent spaces [15], pseudo-Riemannian manifold with recurrent concircular curvature tensor [11], semi-symmetric manifolds [22], pseudo symmetric manifolds [5,24,25], weakly symmetric manifolds [23], projective symmetric manifolds [21], almost pseudo concircularly

Key words and phrases. Conharmonic transformation, conharmonic curvature tensor, pseudo conharmonically symmetric manifold, scalar curvature, perfect fluid spacetime.

2010 Mathematics Subject Classification. Primary: 53B15. Secondary: 53B20, 53B30, 53C15, $53 \mathrm{C} 25$.

Received: May 23, 2017.

Accepted: August 24, 2017. 
symmetric manifolds [9], decomposable almost pseudo conharmonically symmetric manifolds [3], etc.

A non-flat Riemannian or a semi-Riemannian manifold $(M, g)(n>2)$ is said to be a pseudo symmetric manifold [5] if its curvature tensor $R$ satisfies the condition

$$
\begin{aligned}
\left(\nabla_{X} R\right)(Y, Z) W= & 2 A(X) R(Y, Z) W+A(Y) R(X, Z) W+A(Z) R(Y, X) W \\
& +A(W) R(Y, Z) X+g(R(Y, Z) W, X) \rho,
\end{aligned}
$$

where $A$ is a non-zero 1 -form, $\rho$ is a vector field defined by

$$
g(X, \rho)=A(X)
$$

for all $X$ and $\nabla$ denotes the operator of the covariant differentiation with respect to the metric tensor $g$. The 1-form $A$ is called the associated 1-form of the manifold. If $A=0$, then the manifold reduces to a symmetric manifold in the sense of E.Cartan. An $n$-dimensional pseudo symmetric manifold is denoted by $(P S)_{n}$. This is to be noted that the notion of pseudo symmetric manifold studied in particular by Deszcz [10] is different from that Chaki [5]. The notion of weakly symmetric manifolds was introduced by Tamassy and Binh [23]. If the curvature tensor of type $(1,3)$ of a non-flat $n$-dimensional Riemannian manifold $(n>2)$ satisfies the condition

$$
\begin{aligned}
\left(\nabla_{X} R\right)(Y, Z) W= & A(X) R(Y, Z) W+B(Y) R(X, Z) W+D(Z) R(Y, X) W \\
& +E(W) R(Y, Z) X+g(R(Y, Z) W, X) \rho
\end{aligned}
$$

where $\nabla$ denotes the Levi-Civita connection on $(M, g)$ and $A, B, D, E$ and $\rho$ are 1-forms and a vector field respectively, which are non-zero simultaneously, then this manifold is denoted by $(W S)_{n}$. Many authors have been studied weakly symmetric manifolds $[7,8,13,14,16]$, etc.

Conformal transformation of a Riemannian structure is an important object of study in differential geometry. The conharmonic transformation which is a special type of conformal transformations preserves the harmonicity of smooth functions. Such transformation has an invariant tensor which is called the conharmonic curvature tensor. It is easy to verify that this tensor is an algebraic curvature tensor, that is, it possesses the classical symmetry properties of the Riemannian curvature tensor.

Let $\mathrm{M}$ and $\mathrm{N}$ be two Riemannian manifolds with the metrics $g$ and $\bar{g}$, respectively related by

$$
\bar{g}=e^{2 \sigma} g,
$$

where $\sigma$ is a real function. Then $M$ and $N$ are called conformally related manifolds, and the correspondence between $M$ and $N$ is known as conformal transformation [20]. It is known that a harmonic function is defined as a function whose Laplacian vanishes. In generally, the harmonic function is not invariant. In 1957, Ishii obtained the conditions which a harmonic function remains invariant and he introduced the conharmonic transformations as a subgroup of the conformal transformation (1.4) 
satisfying the condition

$$
\sigma_{, h}^{h}+\sigma_{, h}^{h} \sigma_{,}^{h}=0
$$

where comma denotes the covariant differentiation with respect to the metric $g$. A rank-four tensor $H$ that remains invariant under conharmonic transformation of a Riemannian manifold $(M, g)$ is given by

$$
\begin{aligned}
H(X, Y, Z, U)= & R(X, Y, Z, U)-\frac{1}{n-2}[g(Y, Z) S(X, U)-g(X, Z) S(Y, U) \\
& +g(X, U) S(Y, Z)-g(Y, U) S(X, Z)],
\end{aligned}
$$

where $R$ and $S$ denote the Riemannian curvature tensor of type $(0,4)$ defined by $R(X, Y, Z, U)=g(R(X, Y) Z, U)$ and the Ricci tensor of type $(0,2)$, respectively. The curvature tensor defined by (1.6) is known as conharmonic curvature tensor. A manifold whose conharmonic curvature tensor vanishes at every point of the manifold is called conharmonically flat. Thus, this tensor represents the deviation of the manifold from conharmonic flatness. Many authors have been studied the conharmonic curvature tensor, $[1,20]$. The present paper deals with an $n$-dimensional pseudo conharmonically symmetric Riemannian manifold $(M, g)$ (non-conharmonically flat) whose conharmonic curvature tensor $H$ satisfies the condition

$$
\begin{aligned}
\left(\nabla_{X} H\right)(Y, Z, U, V)= & 2 A(X) H(Y, Z, U, V)+A(Y) H(X, Z, U, V) \\
& +A(Z) H(Y, X, U, V)+A(U) H(Y, Z, X, V) \\
& +A(V) H(Y, Z, U, X),
\end{aligned}
$$

where $A$ has the meaning already mentioned in (1.2). Such a manifold is called a pseudo conharmonically symmetric manifold [5] and denoted by $(P C H S)_{n}$. Since the conformal curvature tensor vanishes identically for $n=3$, we assume that $n>3$ throughout the paper.

$L$ denotes the symmetric endomorphism of the tangent space at each point of the manifold corresponding to the Ricci tensor $S$ of type $(0,2)$, that is

$$
g(L X, Y)=S(X, Y)
$$

Let $e_{i}(1 \leq i \leq n)$ be an orthonormal basis of the tangent space at any point of the manifold. From (1.6), we have

$$
H(X, Y)=\sum_{i=1}^{n} H\left(X, e_{i}, e_{i}, Y\right)=\sum_{i=1}^{n} H\left(e_{i}, X, Y, e_{i}\right)=-\frac{r}{n-2} g(X, Y)
$$

and

$$
\sum_{i=1}^{n} H\left(e_{i}, e_{i}, X, Y\right)=\sum_{i=1}^{n} H\left(X, Y, e_{i}, e_{i}\right)=0
$$


where $r$ is the scalar curvature of the manifold. Also, from (1.6) it follows that [18]

$$
\begin{aligned}
& H(X, Y, Z, U)=-H(Y, X, Z, U), \\
& H(X, Y, Z, U)=-H(X, Y, U, Z), \\
& H(X, Y, Z, U)=H(Z, U, X, Y), \\
& H(X, Y, Z, U)+H(X, Z, U, Y)+H(X, U, Y, Z)=0 .
\end{aligned}
$$

We assume that our manifold is $(P C H S)_{n}$. Thus, the relation (1.7) holds.

This paper is organized as follows. Section 2 deals with some properties of $(P C H S)_{n}$. In this section, we find the conditions for the scalar curvature if the Ricci tensor of $(P C H S)_{n}$ is recurrent. In the third section, we give an example for $(P C H S)_{n}$. In the fourth section, we investigate $(\mathrm{PCHS})_{4}$ spacetime. In a perfect fluid $(\mathrm{PCHS})_{4}$ spacetime, some theorems are proved.

\section{Pseudo Conharmonically Symmetric Manifolds}

In this section, firstly, we give some properties of $(P C H S)_{n}$ to use the other section.

Theorem 2.1. The scalar curvature $r$ of $a(P C H S)_{n}$ satisfies the condition

$$
r_{, l}=\left(\frac{2 n+4}{n}\right) A_{l} r
$$

where $A$ is the associated vector field and $r$ is the scalar curvature of $(P C H S)_{n}$.

Proof. We assume that our manifold is $(P C H S)_{n}$. In local coordinates, from (1.7), we have

$$
H_{i j k l, m}=2 A_{m} H_{i j k l}+A_{i} H_{m j k l}+A_{j} H_{i m k l}+A_{k} H_{i j m l}+A_{l} H_{i j k m},
$$

where $A$ is the associated vector field of $(P C H S)_{n}$.

Multiplying (2.1) by $g^{i l} g^{j k}$, we get

$$
H_{, m}=2 A_{m} H+4 A^{l} H_{m l},
$$

where $H_{m l}=g^{i j} H_{i m l j}$ and $H=g^{m l} H_{m l}$.

Contracting (1.9) over $\mathrm{X}$ and $\mathrm{Y}$ and then taking the covariant derivative, we find

$$
H=-\frac{n}{n-2} r \quad \text { and } \quad H_{, m}=-\frac{n}{n-2} r_{, m} .
$$

By putting these equations in (2.2), we finally obtain

$$
r_{, m}=\left(\frac{2 n+4}{n}\right) A_{m} r
$$

Thus, the proof is completed.

Corollary 2.1. In a $(P C H S)_{n}$, if the scalar curvature $r$ is constant then $r$ must be zero.

Proof. In a $(P C H S)_{n}$, we have (2.3). If we assume that $r$ is constant, from (2.3), we get $A_{l} r=0$. Since $A_{l} \neq 0$ in a $(P C H S)_{n}$, thus, $r$ must be zero. 
Theorem 2.2. If a $(P C H S)_{n}$ with non-zero scalar curvature is Ricci-recurrent then the recurrence vector field and the associated vector field of $(\mathrm{PCHS})_{n}$ are related by

$$
\lambda_{l}=\left(\frac{2 n+4}{n}\right) A_{l},
$$

where $\lambda_{l}$ is the recurrence vector field and $A$ is the associated vector field of this manifold.

Theorem 2.3. In $(P C H S)_{n}$ if the conharmonic curvature tensor of a $(P C H S)_{n}$ is Codazzi type, then this manifold must be of zero scalar curvature.

\section{An EXAmple For $(P C H S)_{n}$}

In this section we give an example for $(P C H S)_{n}$ satisfying the conditions (1.6) and (1.7).

We define a Riemannian metric on $\mathbb{R}^{4}$ by the formula [15]

$$
d s^{2}=\varphi\left(d x^{1}\right)^{2}+\left(d x^{2}\right)^{2}+\left(d x^{3}\right)^{2}+2 d x^{1} d x^{4},
$$

where $\varphi$ is a function of $x^{1}, x^{2}, x^{3}$.

In the metric considered, the only non-vanishing components of Christoffel symbols, the curvature tensor and the Ricci tensor are

$$
\begin{gathered}
\Gamma_{11}^{2}=-\frac{1}{2}\left(\varphi_{, 2}+\varphi_{, 3}\right), \quad \Gamma_{11}^{3}=-\frac{1}{2}\left(\varphi_{, 2}+\varphi_{, 3}\right), \\
\Gamma_{11}^{4}=\frac{1}{2} \varphi_{, 1}, \quad \Gamma_{12}^{4}=\frac{1}{2} \varphi_{, 2}, \quad \Gamma_{13}^{4}=\frac{1}{2} \varphi_{, 3}, \\
R_{1221}=\frac{1}{2} \varphi_{, 22}, \quad R_{1231}=\frac{1}{2} \varphi_{, 23}, \quad R_{1331}=\frac{1}{2} \varphi_{, 33}, \quad R_{1321}=\frac{1}{2} \varphi_{, 32}, \\
S_{11}=\frac{1}{2}\left(\varphi_{, 22}+\varphi_{, 33}\right),
\end{gathered}
$$

where "," denotes the partial differentiation with respect to the coordinates.

We consider $\varphi$ as,

$$
\varphi=\left[\left(M_{22}+1\right)\left(x^{2}\right)^{2}+\left(M_{33}+1\right)\left(x^{3}\right)^{2}\right] e^{\left(x^{1}\right)^{2}},
$$

where $M_{22}$ and $M_{33}$ are non-zero constants and satisfy the relation

$$
M_{22}+M_{33}=0 .
$$

In this case, we have the following relations

$$
\begin{aligned}
& \varphi_{, 22}=2\left(M_{22}+1\right) e^{\left(x^{1}\right)^{2}}, \\
& \varphi_{, 33}=2\left(M_{33}+1\right) e^{\left(x^{1}\right)^{2}} .
\end{aligned}
$$


By using (3.2), we find the only non-zero components for $R_{h i j k}$ and $S_{i j}$ as

$$
\begin{gathered}
R_{1221}=\frac{1}{2} \varphi_{, 22}=\left(1+M_{22}\right) e^{\left(x^{1}\right)^{2}}, \quad R_{1331}=\frac{1}{2} \varphi_{, 33}=\left(1+M_{33}\right) e^{\left(x^{1}\right)^{2}}, \\
S_{11}=\frac{1}{2}\left(\varphi_{, 22}+\varphi_{, 33}\right)=2 e^{\left(x^{1}\right)^{2}} .
\end{gathered}
$$

Hence, the only non-zero components of the conharmonic curvature tensor $H_{h i j k}$ are

$$
\begin{aligned}
& H_{1221}=M_{22} e^{\left(x^{1}\right)^{2}}, \\
& H_{1331}=M_{33} e^{\left(x^{1}\right)^{2}},
\end{aligned}
$$

which never vanish. From (3.7), the only non-zero components of the derivative of $H_{\text {hijk }}$ are found as

$$
\begin{aligned}
& H_{1221,1}=2 x^{1} M_{22} e^{\left(x^{1}\right)^{2}}=2 x^{1} H_{1221}, \\
& H_{1331,1}=2 x^{1} M_{33} e^{\left(x^{1}\right)^{2}}=2 x^{1} H_{1331} .
\end{aligned}
$$

Let us consider the associated 1-form as

$$
A_{i}(x)= \begin{cases}\frac{x^{1}}{2}, & \text { for } \mathrm{i}=1 \\ 0, & \text { otherwise }\end{cases}
$$

at any point $x \in V_{n}$.

To verify the relation (1.7) it is sufficient to prove that the equation

$$
\begin{aligned}
& H_{1221,1}=4 A_{1} H_{1221}, \\
& H_{1331,1}=4 A_{1} H_{1331} .
\end{aligned}
$$

By the aid of (3.8) and (3.9), we can easily see that (3.10) is satisfied. The other components of each term of (1.7) vanish identically and the relation (1.7) holds trivially.

Under our assumptions (3.1), (3.3) and (3.4), this manifold is a $(P C H S)_{n}$.

\section{Pseudo Conharmonically Symmetric Spacetimes}

This section deals with certain investigations in general relativity by the coordinate free method of differential geometry. In this method of study, the spacetime of general relativity is a connected four-dimensional semi-Riemannian manifold $\left(M^{4}, g\right)$ with Lorentz metric $g$ with signature $(-,+,+,+)$. The geometry of the Lorentz metric begins with the study of the casual character of vectors of the manifold. It is due to this casuality that the Lorentz manifold becomes a convenient choice for the study of general relativity.

Here, we consider a special type of spacetime which is called pseudo conharmonically symmetric spacetime. A semi-Riemannian four-dimensional pseudo conharmonically 
symmetric manifold may similarly be defined by taking a Lorentz metric $g$ with signature $(-,+,+,+)$.

In this section, we consider a perfect fluid pseudo conharmonically symmetric spacetime having the basic vector field as the timelike velocity vector field $U$ of the fluid, that is

$$
g(U, U)=-1
$$

For a perfect fluid spacetime with the generator $U$ as the flow vector field of the flow and $\mathrm{k}$ as the gravitational constant, we have the Einstein's equation without cosmological constant as

$$
S(X, Y)-\frac{r}{2} g(X, Y)=k T(X, Y)
$$

where $k$ is the gravitational constant, $T$ is the energy momentum tensor of type $(0,2)$ given by

$$
T(X, Y)=(\sigma+p) A(X) A(Y)+p g(X, Y),
$$

with $\sigma$ and $p$ as the energy density and the isotropic pressure of the fluid, respectively, and $A$ as the associated 1-form; $U$ as the generator vector field.

$\mathrm{H}$ is to be noted that the basic geometric features of $(P C H S)_{n}$ manifolds, $n>3$, are also being maintained in the Lorentzian manifold which is necessarily a semiRiemannian manifold. Thus, Theorem 2.1, 2.2, 2.3 are also true for a $(P C H S)_{n}$ spacetime $(n>3)$.

By the aid of (4.2) and (4.3), we can write

$$
S(X, Y)=\left(\frac{r}{2}+k p\right) g(X, Y)+k(\sigma+p) A(X) A(Y) .
$$

Taking a frame field and contracting (4.4) over $X$ and $Y$, we obtain

$$
r=k(\sigma-3 p) \text {. }
$$

In this case, we can state the following theorems.

Theorem 4.1. If the Ricci tensor of a perfect fluid of $(\mathrm{PCHS})_{4}$ spacetime is recurrent then the energy density and the isotropic pressure of this spacetime are proportional.

Proof. Let us assume that our manifold be a perfect fluid of $(\mathrm{PCHS})_{4}$ spacetime. By taking the covariant derivative of (4.4), we get

$$
\begin{aligned}
\left(\nabla_{X} S\right)(Y, Z)= & \left(\frac{d r(X)}{2}+k d p(X)\right) g(Y, Z)+k(d \sigma(X)+d p(X)) A(Y) A(Z) \\
& +k(\sigma+p)\left[\left(\nabla_{X} A\right)(Y) A(Z)+A(Y)\left(\nabla_{X} A\right)(Z)\right] .
\end{aligned}
$$

If our spacetime is Ricci-recurrent then, from Theorem 2.2, we get

$$
\left(\nabla_{X} S\right)(Y, Z)=3 A(X) S(Y, Z) .
$$


Comparing (4.7) with (4.6), we can find that

$$
(d p(X)-3 A(X) p) g(Y, Z)+(d \sigma(X)+d p(X)-3 A(X)(\sigma+p)) A(Y) A(Z)
$$

Setting $Y=Z=U$ in (4.8), we get

$$
d \sigma(X)=3 A(X) \sigma .
$$

From Theorem 2.1, for a $(\mathrm{PCHS})_{4}$ spacetime, we have

$$
d r(X)=3 A(X) r .
$$

By taking the covariant derivative of (4.5) and using (4.9) and (4.10), we obtain

$$
d p(X)=3 A(X) p .
$$

Comparing (4.9) and (4.11), we finally get

$$
\sigma=c p \text {. }
$$

Thus, this completes the proof.

Theorem 4.2. A perfect fluid of $(\mathrm{PCHS})_{4}$ spacetime which is Ricci-recurrent reduces to $\eta$-Einstein spacetime with the condition $\eta=2 \mathrm{kp}$.

Proof. If our manifold which is a perfect fluid $(\mathrm{PCHS})_{4}$ spacetime is Ricci-recurrent then the condition (4.12) holds. If we assume that $c=1$ in (4.12), we find

$$
\sigma=p
$$

which yields, by virtue of (4.5) and (4.13), to

$$
r=-2 k p \text {. }
$$

Comparing (4.4), (4.13) and (4.14), we find

$$
S(X, Y)=2 k p A(X) A(Y) .
$$

Thus, the equation (4.15) shows that this spacetime reduces to $\eta$-Einstein spacetime with the condition $\eta=2 k p$. This completes the proof.

Theorem 4.3. In a perfect fluid of $(\mathrm{PCHS})_{4}$ spacetime, if the energy momentum tensor obeying Einstein's equation without cosmological constant is recurrent then either the associated vector field of this manifold is divergence-free or this manifold reduces to an Einstein spacetime.

Proof. In local coordinates, by taking the covariant derivative of (4.3), we find

$$
T_{i j, l}=\left(\sigma_{, l}+p_{, l}\right) A_{i} A_{j}+(\sigma+p)\left(A_{i, l} A_{j}+A_{i} A_{j, l}\right)+p_{, l} g_{i j} .
$$

We assume that our spacetime is Ricci-recurrent.

By taking the covariant derivative of (4.2) and using (4.7), we find

$$
T_{i j, l}=3 A_{l} T_{i j}
$$


Putting (4.17) in (4.16), changing the indices $j, l$ and subtracting these two equations, we obtain

$$
\begin{aligned}
& 3 A_{l}\left[(\sigma+p) A_{i} A_{j}+p g_{i j}\right]-3 A_{j}\left[(\sigma+p) A_{i} A_{l}+p g_{i l}\right]=p_{, l} g_{i j}-p_{, j} g_{i l}+\left(\sigma_{, l}+p_{, l}\right) A_{i} A_{j} \\
& -\left(\sigma_{, j}+p_{, j}\right) A_{i} A_{l}+(\sigma+p)\left[\left(A_{i, l} A_{j}+A_{i} A_{j, l}\right)-\left(A_{i, j} A_{l}+A_{i} A_{l, j}\right)\right] .
\end{aligned}
$$

Multiplying (4.18) by $g^{i j}$ and multiplying the last equation by $A^{l}$, we find

$$
-9 p=3 A^{l} p_{, l}+(\sigma+p) A_{, l}^{l} .
$$

Since our spacetime is Ricci-recurrent, from Theorem 4.1 and equations (4.11) and (4.19), we get

$$
(\sigma+p) A_{, l}^{l}=0
$$

If we assume that $\sigma=-p$ then, from (4.5) and (4.4), it can be obtained that

$$
S_{i j}=-k p g_{i j}
$$

The equation (4.21) gives us, this spacetime reduces to an Einstein's spacetime. Thus, we must say that $\sigma \neq-p$ and then the vector field generated by the 1 -form $A$ is divergence-free. This completes the proof.

Theorem 4.4. A perfect fluid $(\mathrm{PCHS})_{4}$ spacetime whose conharmonic curvature tensor is Codazzi type represents the equation of state in the radiation era in the evoluation of our universe.

Proof. In a perfect fluid for $(\mathrm{PCHS})_{4}$ spacetime, if the conharmonic curvature tensor is Codazzi type then from Theorem 2.3, we can say that the scalar curvature must be zero. In this case, the equation (4.5) reduces to

$$
\sigma=3 p .
$$

This corresponds the equation of state in the radiation era in the evoluation of our universe. The radiation is the era before the present matter dominated era. Thus, the proof is completed.

\section{Conclusion}

General relativity takes the form of field equations, describing the curvature of spacetime and the distribution of matter throughout spacetime. The effects of matter and spacetime on each other are what we perceive as gravity.

The theory of the spacetime continuum already existed, but under general relativity Einstein was able to describe gravity as bending of spacetime geometry. Einstein defined a set of field equations, which represented the way that gravity behaved in response to matter in spacetime. These field equations could be used to represent the geometry of spacetime that was at the heart of the theory of general relativity. Because spacetime curve, the objects moving through space would follow the "straightest" path 
along the curve, which explains the motion of the planets. "In general relativity the matter content of the spacetime is described by the energy momentum tensor $\mathrm{T}$ which is to be determined from physical considerations dealing with the distribution of matter and energy. Since the matter content of the universe is assumed to behave like a perfect fluid in the standard cosmological models, the physical motivation for studying Lorentzian metric defined on a suitable four dimensional manifold M. Relativistic fluid models of considerable interest in several areas of astrophysics, plasma physics and nuclear physics, Theories of relativistic stars (which would be models for supermassive stars) are also based on relativistic fluid models. The problem of accretion onto a neutron stars or a black hole is usually set in the framework of relativistic fluid models" [17].

"The physical motivation for studying various types of spacetime models in cosmology is to obtain the information of different phases in the evolution of the universe, which may be classified into three phases, namely, the initial phase, the intermediate phase and the final phase. The initial phase is just after the Bing Bang when the effects of both viscosity and heat flux were quite pronounce. The intermediate phase is that when the effect of viscosity was no longer significant but the heat flux was till not negligible. The final phase, which extends to the present state of the universe when both the effects of viscosity and heat flux have become negligible and the matter content of the universe may be assumed to be perfect fluid" [19]. As it is well known, symmetric spaces play an important role in differential geometry. This paper deals with pseudo conharmonically symmetric spaces. The conharmonic transformation which is a special type of conformal transformations preserves the harmonicity of smooth functions. Since the conharmonic curvature tensor which is an invariant under the conharmonic transformation is an algebraic curvature tensor, it possesses the classical symmetry properties of the Riemannian curvature. In this paper, we consider the pseudo conharmonically symmetric curvature tensor in a spacetime and we prove some theorems about the energy density and the isotropic pressure. In addition, we prove that in a perfect fluid pseudo conharmonically symmetric spacetime if the energy momentum tensor obeying Einstein's equation without cosmological constant is recurrent then either the associated vector field of this manifold is divergence-free or this manifold reduces to Einstein.

\section{REFERENCES}

[1] D. B. Abdussatter, On conharmonic transformations in general relativity, Bull. Calcutta Math. Soc. 41 (1966), 409-416.

[2] T. Adati and T. Miyazawa, On a Riemannian space with recurrent conformal curvature, Tensor (N.S.) 18 (1967), 348-354.

[3] H. Bagdatli Yilmaz, On decomposable almost pseudo conharmonicaly symmetric manifolds, Acta Univ. Palack. Olomuc. Fac. Rerum Natur. Math. 51(1) (2012), 111-124.

[4] E. Cartan, Surune classe remarquable d' espaces de Riemannian, Bull. Soc. Math. France 54 (1926), 214-264. 
[5] M. C. Chaki, On pseudo symmetric manifolds, An. Stiint. Univ. Al. I. Cuza Iasi. Mat. 33 (1987), 53-58.

[6] M. C. Chaki and B. Gupta, On conformally symmetric spaces, Indian J. Math. 5 (1963), 113-295.

[7] U. C. De and S. Bandyopadhyay, On weakly symmetric Riemannian spaces, Publ. Math. Debrecen 54 (1999), 371-381.

[8] U. C. De and S. Bandyopadhyay, On weakly symmetric spaces, Acta Math. Hungar. 83 (2000), 205-212.

[9] U. C. De and S. Mallick, On almost pseudo concircularly symmetric manifolds, The Journal of Mathematics and Computer Science 4(3) (2012), 317-330.

[10] R. Deszcz and W. Grycak, On some class of warped product manifolds, Bull. Inst. Math. Acad. Sin. 15 (1987), 311-322.

[11] K. Olszak and Z. Olszak, On pseudo-Riemannian manifolds with recurrent concircular curvature tensor, Acta Math. Hungar. 137(1-2) (2012), 64-71.

[12] B. O' Neill, Semi-Riemannian Geometry with Applications to the Relativity, Academic Press, New York-London, 1983.

[13] M. Prvanovic, On weakly symmetric Riemannian manifolds, Publ. Math. Debrecen 46 (1995), 19-25.

[14] M. Prvanovic, On totally umbilical submanifolds immersed in a weakly symmetric Riemannian manifolds, Izv. Vyssh. Uchebn. Zaved. Mat. 6 (1998), 54-64.

[15] W. Roter, On conformally symmetric Ricci-recurrent spaces, Colloq. Math. 31 (1974), 87-96.

[16] A. A. Shaikh, I. Roy and S. K. Hui, On totaly umbilical hypersurfaces of weakly conharmonically symmetric spaces, Global Journal of Science Frontier Research 10(4) (2010), 28-31.

[17] A. A. Shaikh and K. K. Baishya, On concircular structure spacetimes, Journal of Mathematics and Statistics 1 (2005), 129-132.

[18] A. A. Shaikh and S. K. Hui, On weakly conharmonically symmetric manifolds, Tensor (N.S) 70 (2008), 119-134.

[19] A. A. Shaikh and S. K. Hui, On pseudo cyclic Ricci symmetric manifolds admitting semisymmetric metric connection, Scientia 20 (2010), 73-80.

[20] S. A. Siddiqui and Z. Ahsan, Conharmonic curvature tensor and the spacetime of general relativity, Bull. Calcutta Math. Soc. 41 (1966), 409-416.

[21] G. Soos, Uber die geodatischen Abbildungen von Riemannschen Raumen auf projektiv symmetrische Remannsche Raume, Acta Mathematica Academiae Scientiarum Hungaricae 9 (1958), 359-361.

[22] Z. I. Szabo, Structure theorems on Riemannian spaces satisfying $R(X, Y) R=0$, J. Differential Geom. 17 (1982), 531-582.

[23] L. Tamassy and T. Q. Binh, On weakly symmetric and weakly projectively symmetric Riemannian manifolds, Colloquia Mathematica Societatis Janos Bolyai 56 (1989), 663-670.

[24] F. Özen and S. Altay, On weakly and pseudo symmetric Riemannian spaces, Indian J. Pure Appl. Math. 33(10) (2001), 1477-1488.

[25] F. Özen and S. Altay, On weakly and pseudo concircular symmetric structures on a Riemannian manifold, Acta Univ. Palack. Olomuc. Fac. Rerum Natur. Math. 47 (2008), 129-138.

[26] A. G. Walker, On Ruse's space of recurrent curvature, Proc. Lond. Math. Soc. 52 (1951), 36-64. 
${ }^{1}$ Department of Mathematics, ISTANBUL TECHNICAL UNIVERSITY, 34469 IstanBul, TURKEY

Email address: fozen@itu.edu.tr

${ }^{2}$ Department of Mathematics, Piri ReIs University,

ISTANBUL, TURKEY

Email address: aytasci@pirireis.edu.tr 